\title{
Effect of cluster scavenging on homogeneous nucleation
}

\author{
G. Shi and J. H. Seinfeld \\ California Institute of Technology, Pasadena, California 91125
}

(Received 2 August 1989; accepted 13 September 1989)

\begin{abstract}
A closed-form expression for the effect of cluster scavenging on the rate of homogeneous nucleation of a vapor in the presence of continuum regime particles is obtained by solving the kinetic equation of nucleation by the method of singular perturbation. The reduction in nucleation rate of a condensing species at a given supersaturation is shown to be dependent largely on the number concentration, the size of the sink particles, and the molecular number concentration of the background gas. The reduction in the rate of nucleation due to the cluster scavenging by transition regime particles is also discussed.
\end{abstract}

\section{INTRODUCTION}

Homogeneous nucleation occurs when a vapor becomes sufficiently supersaturated that critical sized clusters form at a detectable rate. Preexisting or nucleated particles act to scavenge vapor molecules as well as clusters and therefore to depress the rate of homogeneous nucleation below that in their absence. When the scavenging particles have radii much smaller than the mean free paths of the vapor molecules and clusters, the so called free molecule regime, the depression in the overall nucleation rate is a function of saturation ratio and the ratio of the surface area concentration of scavenging particles to that of the monomer. ${ }^{1}$ For scavenging particles with radii much larger than the mean free paths, the so called continuum regime, the diffusion of monomers and clusters to the particle leads to spatial inhomogeneities in the neighborhood of each particle. Because the effectiveness of the scavenging depends on the particle size and number concentration, the rate of homogeneous nucleation occurring in the bulk system will depend on the size and number concentration of scavenging particles in addition to the saturation ratio.

Pesthy $e t a l_{.}^{2}$ and Stern $e t a l .^{3}$ considered the effect of monomer depletion by scavenging particles on the rate of homogeneous nucleation of a vapor but neglected cluster scavenging. McGraw and McMurry ${ }^{4}$ used dimensional arguments to show that cluster diffusion in the neighborhood of a $1 \mu \mathrm{m}$ diameter particle cannot usually be neglected.

The goal of this paper is to derive an expression for the influence of cluster scavenging on the rate of homogeneous nucleation of a vapor in presence of continuum regime particles. The result will be obtained in a form showing explicitly the deviation from the rate of homogeneous nucleation considering only monomer depletion.

\section{BASIC EQUATIONS}

The dynamic behavior of a spectrum of growing and evaporating clusters in a spatially nonuniform system is governed by

$$
\begin{aligned}
\frac{d f_{g}}{d t}-D_{g} \nabla^{2} f_{g}= & \frac{1}{2} \sum_{k=1}^{g} \beta_{g-k, k} f_{g-k} f_{k}-f_{g} \sum_{k=1}^{\infty} \beta_{g, k} f_{k} \\
& +\sum_{k=g+1}^{\infty} \alpha_{k-g, g}^{k} f_{k}-\frac{1}{2} f_{g} \sum_{k=1}^{g-1} \alpha_{g-k, k}^{g},
\end{aligned}
$$

where $f_{g}$ is the concentration of clusters containing $g$ monomers, $D_{g}$ is the diffusion coefficient of the $g$-sized cluster, $\beta_{i, j}$ is the probability of formation of a cluster of size $g$ by the collision of $i$ - and $j$-sized clusters, and $\alpha_{i, j}^{g}$ is the probability that a cluster of size $g$ will fission into clusters of sizes $i$ and $j$. Equation (1) has also been written in a slightly different context by other authors. ${ }^{5}$

Defining the cluster flux arising by addition or evaporation only of individual monomers, i.e., neglecting clustercluster coagulation as in classical nucleation theory, we can introduce the flux

$$
J_{g}=\beta_{g-1,1} f_{g-1}-\alpha_{g} f_{g}
$$

and Eq. (1) can be rearranged as

$$
\frac{d f_{g}}{d t}=J_{g}-J_{g+1}+D_{g} \nabla^{2} f_{g} \text {. }
$$

As a standard practice in nucleation theory, detailed balancing is used to relate the $\alpha_{i j}$ to $\beta_{i j}$ and the equilibrium (with respect to the monomer) concentration of clusters. As a result, we have

$$
J_{g}=-\beta_{1, g} n_{g}\left(f_{g+1} / n_{g+1}-f_{g} / n_{g}\right),
$$

where $n_{g}$ is the equilibrium cluster size distribution corresponding to the monomer $n_{1}$ (not to the bulk liquid) and $\beta_{1, g}$ is the product of the surface area of cluster containing $g$ monomers with the rate, based on the Maxwell-Boltzmann distribution of velocities, at which a unit surface area is hit by monomers. $\beta_{1, g}$ is equivalent to $\beta(g, t)$ given below.

Within the limits of the classical nucleation theory of Frenkel, Becker-Döring, and Zeldovich, ${ }^{6} J_{g}$ may be replaced by $J(g, t)$ which is the cluster flux defined in the continuous cluster size space and its relationship with the continuous cluster size distribution, $f(g, t)$, immediately follows as

$$
\frac{\partial f(g, \mathbf{r}, t)}{\partial t}=-\frac{\partial J(g, \mathbf{r}, t)}{\partial g}+D(g) \nabla^{2} f(g, \mathbf{r}, t),
$$

where $\mathbf{r}$ is the spatial coordinate vector and

$$
J(g, \mathbf{r}, t)=-\beta(g, \mathbf{r}, t) n(g, \mathbf{r}, t) \frac{\partial}{\partial g} \frac{f(g, \mathbf{r}, t)}{n(g, \mathbf{r}, t)} .
$$

One boundary condition on Eq. (5) expresses the fact that the equilibrium and steady-state monomer concentrations are equal, 


$$
f(1, \mathbf{r}, t)=n(1, \mathbf{r}, t) .
$$

The second boundary condition on $g$ is that there are no particles of infinite size

$$
f(\infty, \mathbf{r}, t)=0 .
$$

Two more boundary conditions on the spatial coordinate are needed in addition to the above two boundary conditions on g. Also in Eq. (6),

$$
\beta(g, \mathbf{r}, t)=f(1, t) s_{1} g^{2 / 3}\left(k T / 2 \pi m_{1}\right)^{1 / 2}
$$

with $s_{1}, m_{1}$ the surface area and mass of the monomer and the diffusivity of a $g$-mer with its size much smaller than the mean free path of the background gas, is given by

$$
D(g)=\frac{1.105 g^{-2 / 3}}{s_{1} n_{a}}\left(\frac{\pi k T}{2 m_{1}}\right)^{1 / 2},
$$

where $n_{a}$ is the molecular number concentration of the background gas. The classical equilibrium cluster distribution is

$$
n(g)=n(1) \exp \left(g \ln S-\theta g^{2 / 3}\right),
$$

where $\theta$ is a dimensionless surface tension

$$
\theta=s_{1} \sigma / k T
$$

and where the saturation ratio $S=n(1) / n_{\mathrm{eq}}(1), \sigma$ is the surface tension of the planar liquid, and $n_{\mathrm{eq}}$ (1) the equilibrium number concentration of the monomer.

Equation (5) is solved in the steady-state spatially homogeneous case to obtain the classical nucleation rate. ${ }^{6}$ In the present case the inhomogeneities of monomer and clusters in the neighborhood of each scavenging particle make the rate of homogeneous nucleation a function of spatial position. The procedure used in Ref. 1 can only be followed after obtaining an effective kinetic equation for the cluster balances in which the effect of scavenging is represented as a volumetric sink term. The first necessary step lies in the construction of a realistic procedure of averaging to obtain the effective governing equation. The influence of cluster scavenging will be presented as a ratio of the rate of homogeneous nucleation considering monomer depletion and cluster scavenging and that considering only monomer depletion.

\section{EFFECTIVE EQUATION AND GROWTH RATE}

\section{A. Effective equation}

To account for the potential many-body effect, a method of averaging over the configurational ensemble of the particle sinks in the system must be established. In a system without a phase transition such as in diffusion-controlled reactions, the effective equations are obtained through truncation of the chain of equations for the many-particle distribution functions with the aid of the cluster expansion method, scaling expansion approach, and other standard approaches of statistical mechanics, ${ }^{7-14}$ which rely on expansions in the smallness of the spatial gradients or sink concentration or in the slowness of temporal change. A more tractable averaging makes use of effective medium theories. Averaging Eq. (5) over the spherical volume elements containing many particles but of the same order as the diffusion length scale $l$, we have

$$
\frac{\partial\langle f(\mathbf{g}, \mathbf{r}, t)\rangle}{\partial t}=-\left\langle\frac{\partial J(g, \mathbf{r}, t)}{\partial g}\right\rangle+\left\langle D(g) \nabla^{2} f(g, \mathbf{r}, t)\right\rangle,
$$

where $\langle f(g, \mathbf{r}, t)\rangle$ is the cluster concentration averaged in the indicated manner, i.e.,

$$
\langle f(g, \mathbf{r}, t)\rangle \equiv \frac{1}{V} \iiint_{V} f(g, \mathbf{r}, t) d v .
$$

Equation (13) describes the time variation of the average cluster concentration $\langle f(g, \mathbf{r}, t)\rangle$ as the result of nucleation as well as capture and evaporation by the sink particles. The first two terms in the above equation are obtained naturally from Eq. (5). The decomposition of the last term on the right-hand side of Eq. (13) requires more attention. In the case of a macroscopically spatially homogeneous system, the spatial coordinate is excluded from any averaged quantity. In addition, we can assume div $[-D(g) \nabla\langle f\rangle]=0$, i.e., inhomogeneties over the scale of $l$ are negligible. With allowance for the boundary condition Eq. (8), we have

$$
\begin{aligned}
\left\langle D(g) \nabla^{2} f(g, t)\right\rangle & =\left.\frac{4 \pi D(g)}{V} \sum_{i=1}^{N} R_{i}^{2} \nabla f\right|_{\mathrm{r}=\mathrm{r}_{\mathrm{i}}+\mathrm{R}_{\mathrm{i}}} \\
& =\left.4 \pi D(g) \rho \int_{R^{*}}^{\infty} R^{2} \nabla f\right|_{\mathrm{r}=\mathrm{r}_{\mathrm{i}}+\mathrm{R}_{\mathrm{i}}} P(R) d R,
\end{aligned}
$$

where $P(R)$ is the probability size distribution of the existing particles which is assumed to be independent of position, $R$ * is a lower limit for particle radii in the diffusion regime, $N$ is the total number of particles in the volume $V$, and $\rho$ is the number density of the particles. In deriving the above expression, Gauss's theorem is used to reduce the volume integral to an integral over all particle surfaces and over the outer boundary of the volume $V$ which, subject to the condition of spatial inhomogeneity, results in the summation of all fluxes over all the sinks in the volume $V$ and diffusion over the length scale $l$ which is neglected in the present case.

To find $\nabla f$, in principle, it is necessary to solve $\mathrm{Eq} .(5)$ in the vicinity of each particle with appropriate boundary conditions. However some reasonable assumptions based on considering the time scale of each physical process [each term of Eq. (5) ] enable us to determine $\nabla f$. We are assuming that the characteristic time to establish the steady-state cluster distribution is short compared to changes in the saturation ratio $S$, the term $\partial f / \partial t$ can be omitted from Eq. (5). Secondly, the characteristic relaxation time for establishment of the steady-state spatial distribution is $\tau_{d} \sim d^{2} / D(g)$, where $d$ is the characteristic length. The steady-state cluster distribution over $g$ is established in a time $\tau_{n} \sim g / \beta(g)$. For the condition $\tau_{n} / \tau_{d} \gg 1$ to hold requires that $p_{\text {eq }} \ll 4.441 g^{-1 / 3} S^{-1}$ at $T=298 \mathrm{~K}$ and 1 atm of the pressure of the background gas and $d=10^{-4} \mathrm{~cm} . p_{\mathrm{eq}}$ is in Torr. For $S=10$ to 1000 and $g=50$, the critical range for $p_{\text {eq }}$ is 0.12 $10^{-4}$ Torr. Therefore for values of $p_{\text {eq }}$ in the range we are most interested in (organic vapors and other particle-forming species), $\tau_{d} \ll \tau_{n}$ holds and the nucleation term in Eq. (5) can be omitted for the present purpose of estimating $\nabla f$ in Eq. (15). By also neglecting the Stefan flow and the effect of latent heat associated with condensation, determining $\nabla f$ in Eq. (15) reduces to solving the diffusion equation 


$$
\nabla^{2} f(g, \mathbf{r})=0
$$

with appropriate boundary conditions.

It should be noted that the similar arguments were also made in the work of Pesthy et al. ${ }^{2}$

\section{B. Growth rate by $g$-mer scavenging}

The cluster concentration field around a particle with its center at the origin takes the following form from solving Eq. (16) in the case where the growth rate is diffusion limited

$$
f(g, r)=f(g, \infty)+\frac{R}{r}[f(g, R)-f(g, \infty)],
$$

where $f(g, \infty)$ is the cluster size distribution at a position far from the particle. By substituting Eq. (17) into Eq. (13) and using Eq. (15), we obtain

$$
\frac{\partial\langle f(g, t)\rangle}{\partial t}=-\left\langle\frac{\partial J(g, t)}{\partial g}\right\rangle-4 \pi \int_{R^{*}}^{\infty} \rho R^{2} \frac{d R}{d t} P(R) d R,
$$

where

$$
\frac{d R}{d t}=\left.D(g) \nabla f\right|_{r=R}=\frac{D(g)}{R}[f(g, \infty)-f(g, R)]
$$

is the partial growth rate, i.e., the contribution to the growth rate of a particle of radius $R$ as a result of $g$-sized cluster scavenging.

At a nonzero concentration of sink particles, Eq. (17) has to be significantly modified. The reason that the modification is qualitatively significant even for dilute systems is that the steady-state concentration field surrounding a particle is of long range. One of the boundary conditions used in obtaining Eq. (17) is $f(g, r) \rightarrow f(g, \infty)$ as $r \rightarrow \infty$, which is only applicable in the limit of zero sink concentration. At finite concentration of sink particles, the full many-body problem can reduce to the consideration of an isolated particle in an effective medium if a mean-field approximation is invoked. In the mean-field picture, a single particle grows as a result of the diffusion-limited flux of monomers and clusters from the effective medium. We can specify a boundary condition at $l$ for Eq. (16), i.e.,

$$
\left.f\right|_{r=l}=f(g, l) \text {. }
$$

Accordingly, the effect of sink particles of different sizes within a volume of characteristic length $l$ is represented by the profile around a particle of average size which is given by

$$
f(g, r)=f(g, l)+\frac{\bar{R}}{r} \frac{l-r}{l-\bar{R}}[f(g, \bar{R})-f(g, l)] .
$$

Since the characteristic length scale of a diffusion process is $\sqrt{D(g) t_{c}}$, we can take $l \propto \sqrt{D(g) t_{c}}$. The characteristic time scale $t_{c}$ for removal of clusters from this characteristic volume is given by

$$
t_{c}=\frac{1}{4 \pi D_{g} \bar{R} \rho},
$$

where $\bar{R}$ is the average particle radius. Thus the length scale $l$ is given by

$$
l=\text { const } \frac{1}{\sqrt{4 \pi \bar{R} \rho}}
$$

which is seen to be inversely proportional to the product of the number concentration of particles and their average radius. In the following $P(R)$ is taken to equal 1 for convenience, i.e., $R=\bar{R}$.

Consistent with our approximations above, we can relate $f(g, l)$ to $\langle f(g, r)\rangle$ by averaging Eq. (21)

$$
\begin{aligned}
\langle f(g)\rangle \equiv\langle f(g, r)\rangle & =\frac{1}{V} \iiint_{V} f(g, r) d v \\
& =f(g, l)+\alpha[f(g, R)-f(g, l)],
\end{aligned}
$$

which turns out to be independent of the spatial coordinate as desired and where

$$
\begin{aligned}
\alpha= & \left\{\frac{R}{l}\left[\frac{1}{2}\left(1+\frac{R}{l}\right)-\left(\frac{R}{l}\right)^{2}\right]\right\} \\
& \times\left\{\left(1-\frac{R}{l}\right)\left[1+\frac{R}{l}+\left(\frac{R}{l}\right)^{2}\right]\right\}^{-1} .
\end{aligned}
$$

Thus

$$
f(g, l)=\frac{\langle f(g)\rangle}{1-\alpha}-\frac{\alpha}{1-\alpha} f(g, R) .
$$

In this case, we have

$$
\frac{d R}{d t}=\frac{D(g)}{R}[\langle f(g, r)\rangle-f(g, R)] \frac{1}{1-\alpha} \frac{l}{l-R},
$$

which reduces to Eq. (19) in the limit of $R / l \rightarrow 0$. By neglecting second- and higher-order powers in $R / l$, i.e., in the limit of small but finite sink volume fraction, Eq. (27) becomes

$$
\frac{d R}{d t}=\frac{D(g)}{R}[\langle f(g)\rangle-f(g, R)][1+R \sqrt{4 \pi \rho \bar{R}}],
$$

which is in the same form as obtained by the method of multiple scattering ${ }^{13}$ (the constant appearing in $l$ is taken as $3 / 2$ ). Also, the rate of diffusion-controlled reaction in the presence of random traps can be obtained from the above result which is in agreement with the commonly accepted expression for the first-order concentration dependence of the reaction rate. ${ }^{7-14}$ In particular, the result is numerically in agreement with that obtained by Mattern and Felderhof ${ }^{11}$ up to a volume fraction $\phi\left(=4 / 3 \pi \rho R^{3}\right)$ about 0.15 .

In the following, we will obtain the nucleation rate in a field of nonzero volume fraction of sink particles using the growth rate given by Eq. (28).

\section{STEADY-STATE SIZE DISTRIBUTION AND NUCLEATION RATE}

Assuming the sink particle size distribution is sufficiently narrow as to be characterized by a single average radius $R$, with the growth law obtained in the last section, Eq. (28), the governing equation for the cluster size distribution, Eq. (13) takes the form

$$
\begin{aligned}
& \frac{\partial\langle f(g, t)\rangle}{\partial t}=-\left\langle\frac{\partial J(g, t)}{\partial g}\right\rangle \\
&-4 \pi D(g) \rho\left[\langle f(g, t)\rangle-f_{R}(g, t)\right] \\
& \times R(1+R \sqrt{4 \pi R \rho}) \\
&\langle f(1, t)\rangle=\langle n(1, t)\rangle, \quad\langle f(\infty, t)\rangle=0
\end{aligned}
$$


where $f_{R}(g, t)$ is $f(g, t)$ at a particle surface.

Our goal is to obtain the effect of cluster $(g \geqslant 2)$ scavenging by continuum regime particles on the nucleation rate. Previous work has addressed the effect of monomer scavenging only. ${ }^{2,3}$ Because of the strong dependence of the nucleation rate on $S$, nucleation will be suppressed in the immediate vicinity of a particle where the monomer is being removed. Moreover, the strong nonlinear dependence of the nucleation rate on $S$ invalidates the equality between $J(\langle S\rangle)$ and $\langle J(S)\rangle$. Representing the effect of cluster scavenging as a mean volumetric sink term in the kinetic equation of nucleation [Eq. (29)] and thereby obtaining the overall nucleation rate based on the spatial average saturation ratio $\langle S\rangle$ is our main approximation. A similar approximation was used in previous work ${ }^{15}$ and was assessed later by comparison with the cell model. ${ }^{16}$ It was found that if the volume fraction $\phi$ of preexisting aerosol is less than about $10^{-6}$, $J\langle S\rangle$ will be indistinguishable from $\langle J(S)\rangle$. Thus our results obtained below are limited to $\phi<10^{-6}$.

Introducing the new independent variables $y=\langle f(g)\rangle /\langle n(g)\rangle, x=g / g_{*}$, where

$$
g_{*}=\left(\frac{2 \theta}{3 \ln \langle S\rangle}\right)^{3}
$$

Eq. (29) under steady-state condition becomes

$\epsilon^{2} \frac{d^{2} y}{d x^{2}}+\left(\frac{2}{3 x} \epsilon^{2}+6\left(1-x^{-1 / 3}\right)\right) \frac{d y}{d x}-2 \eta x^{-4 / 3} y=0$,

where $\epsilon=\delta / g_{*}$, the width of the critical region $\delta=3 g_{*}^{2 / 3} \theta^{-1 / 2}$. We can specify $f_{R}(g)=0$ and $\eta$ in Eq. (31) is given by

$$
\begin{aligned}
\eta & =\frac{4 \pi \rho R D\left(g_{*}\right)(1+R \sqrt{4 \pi R}) \delta^{2}}{\beta\left(g_{*}\right)} \\
& =\frac{19.884 \pi^{2} \rho R(1-\sqrt{3 \phi})}{\theta s_{1}^{2} n_{1} n_{a}} .
\end{aligned}
$$

The dimensionless parameter $\eta$ represents the ratio of the flux of clusters to preexisting particles to the flux in the cluster size space over the Gibbs energy barrier at $g=g_{*}$. In the absence of preexisting particles $\eta=0$. An increasing level of preexisting particles is reflected in an increasing value of $\eta$. Equation (31) together with the boundary conditions

$$
y\left(1 / g_{*}\right)=1, \quad y(\infty)=0
$$

can now be solved by the method of singular perturbation. Since the dominant term of $d y / d x$ changes sign at $x=1$ in the interval $\left[1 / g_{*}, \infty\right]$, we expect a transition layer at $x=1$. Thus there are two outer solutions: a $y_{\text {out }}^{l}$ that satisfies the left boundary condition at $x=1 / g_{*}$ and a $y_{\text {out }}^{r}$ that satisfies the right boundary condition at $x \rightarrow \infty$. The outer solution is expected to be valid everywhere except in a small region (inner region) around $x=1$.

The outer solutions are valid far from the critical region and must satisfy the boundary conditions

$$
y_{0}^{l}\left(1 / g_{*}\right)=1, \quad y_{n}^{l}\left(1 / g_{*}\right)=0, \quad n \geqslant 1
$$

and

$$
y_{n}^{r}(\infty)=0, \quad n \geqslant 0 .
$$

The outer solutions are

$$
y_{\mathrm{out}}^{l}=\left(\frac{x^{-1 / 3}-1}{g_{*}^{1 / 3}-1}\right)^{\eta}
$$

and

$$
y_{\text {out }}^{r}=0 \text {. }
$$

As expected, the outer solution is not valid near $x=1$, so a boundary layer at $x=1$ exists. Since the thickness of this boundary layer is $\epsilon$, we introduce the inner variables $X=(x-1) / \epsilon$ and $Y_{\text {in }}(X) \equiv y_{\text {in }}(x)$. In terms of these variables, Eq. (31) becomes

$$
\begin{aligned}
\frac{\epsilon}{3} \frac{d Y_{\text {in }}}{d X}+\frac{1}{2}(1+\epsilon X) \frac{d^{2} Y_{\text {in }}}{d X^{2}} \\
+3 \frac{(1+\epsilon X)}{\epsilon}\left[1-(1+\epsilon X)^{-1 / 3}\right] \frac{d Y_{\text {in }}}{d X} \\
\quad=\eta(1+\epsilon X)^{-1 / 3} Y_{\text {in }}, \quad Y_{\text {in }}(\infty)=0 .
\end{aligned}
$$

Representing $Y_{\text {in }}$ as a perturbation series in powers of $\epsilon$, Eq. (38) can be converted into a sequence of second-order differential equations. We solve the leading equation

$$
\frac{1}{2} \frac{d^{2} Y_{0}}{d X^{2}}+X \frac{d Y_{0}}{d X}=\eta Y_{0}
$$

to obtain the leading-order inner solution as

$$
Y_{0}=\frac{1}{2} A i^{\eta} \operatorname{erfc}(X)+\frac{1}{2} B i^{\eta} \operatorname{erfc}(-X),
$$

where $i^{\eta}$ erfc $(X)$ is a repeated error function and the constant $A$ has to be determined by asymptotically matching the outer and inner solutions. The match consists of requiring that the intermediate limits $[\epsilon \rightarrow 0+, x \rightarrow 1-, X \rightarrow-\infty]$ of the inner and outer solutions agree. The leading-order match gives

$$
A=\left(\frac{\epsilon}{3}\right)^{\eta} \Gamma(\eta+1)\left(g_{*}^{1 / 3}-1\right)^{-\eta}, \quad B=0 .
$$

However, to determine the nucleation rate, we are most interested in the cluster size distribution near the critical size which is given by the inner solution (leading order in $\epsilon$ )

$y(g)=\frac{1}{2}\left(\frac{\epsilon}{3}\right)^{\eta} \Gamma(\eta+1)\left(g_{*}^{1 / 3}-1\right)-{ }^{\eta} i^{\eta} \operatorname{erfc}\left(\frac{g-g_{*}}{\delta}\right)$.

In the limit of no existing sinks, i.e., $\eta \rightarrow 0$, the cluster size distribution in the critical region is ${ }^{17}$

$$
f(g)=\frac{1}{2} n(g) \operatorname{erfc}\left(\frac{g-g_{*}}{\delta}\right) .
$$

The rate of nucleation in the presence of preexisting continuum regime particles is

$$
\begin{aligned}
\left\langle J\left(g_{*}\right)\right\rangle & =-\left.\beta\left(g_{*}\right) n\left(g_{*}\right) \frac{\partial}{\partial g} \frac{f(g)}{n(g)}\right|_{8=g_{*}} \\
& =\left\langle J_{0}\right\rangle\left(\frac{\epsilon}{6\left(g_{*}^{1 / 3}-1\right.}\right)^{\eta} \sqrt{\pi} \frac{\Gamma(\eta+1)}{\Gamma\left(\eta / 2+\frac{1}{2}\right)},
\end{aligned}
$$

where $\left\langle J_{0}\right\rangle$ is the rate of homogeneous nucleation of a vapor taking into account the effect of monomer depletion but neglecting cluster scavenging. In the absence of any preexisting particles, $\left\langle J\left(g_{*}\right)\right\rangle=\left\langle J_{0}\left(g_{*}\right)\right\rangle$. 
From Eq. (27), the spatial average saturation ratio for $\phi \ll 1$ can be approximated as

$$
\langle S\rangle \approx(1-\alpha) S+\alpha \approx\left(1-\frac{3}{4} \sqrt{3 \phi}\right) S+\frac{3}{4} \sqrt{3 \phi}
$$

by assuming that $f(1, l)$ equals the monomer number concentration in the absence of existing particles and neglecting the Kelvin effect.

\section{DISCUSSION}

The major significance of scavenging is its effect on the steady-state nonequilibrium cluster size distribution and consequently on the rate of nucleation. Such distributions are presented in Fig. 1 (a) in the presence of cluster scavenging [Eqs. (36) and (42)] and in its absence [Eq. (43)] for $\eta=1$. Not surprisingly, in comparison to the classical distribution, scavenging by particles greatly depletes the nonequilibrium number concentration of clusters. Since the nucleation rate is determined by the rate of collision of the monomer with critical sized clusters, a decrease in the number concentration of critical sized clusters results in a decrease in the rate of nucleation. Also shown in Fig. 1(a) is the thermodynamic equilibrium cluster size distribution $n(g)$. It can be seen that the deviation of the steady-state distribution from the equilibrium is greater in the presence of cluster scavenging. In Fig. 1(b), the normalized cluster size distributions are shown. In the absence of scavenging parti-
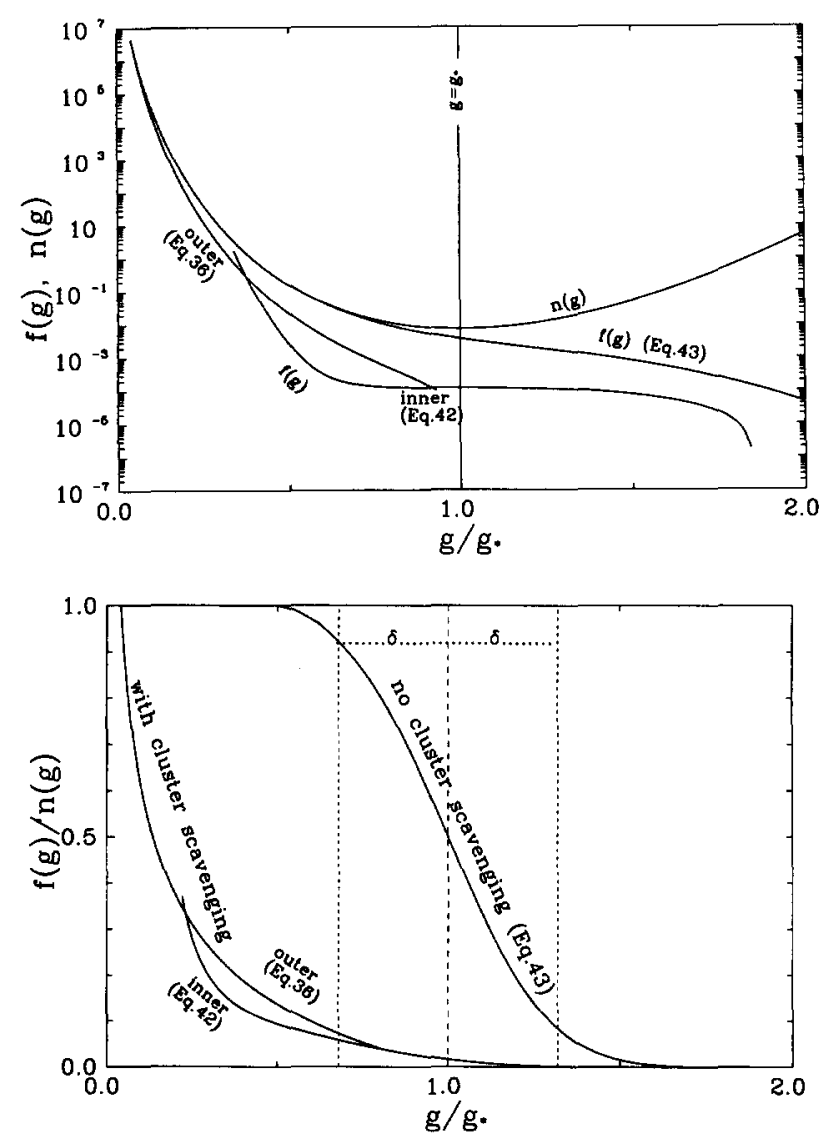

FIG. 1. The steady-state nonequilibrium cluster size distributions in (a) the case of cluster scavenging [Eqs. (36) and (42)-left outer and inner solutions] and (b) in its absence [Eq. (43)].

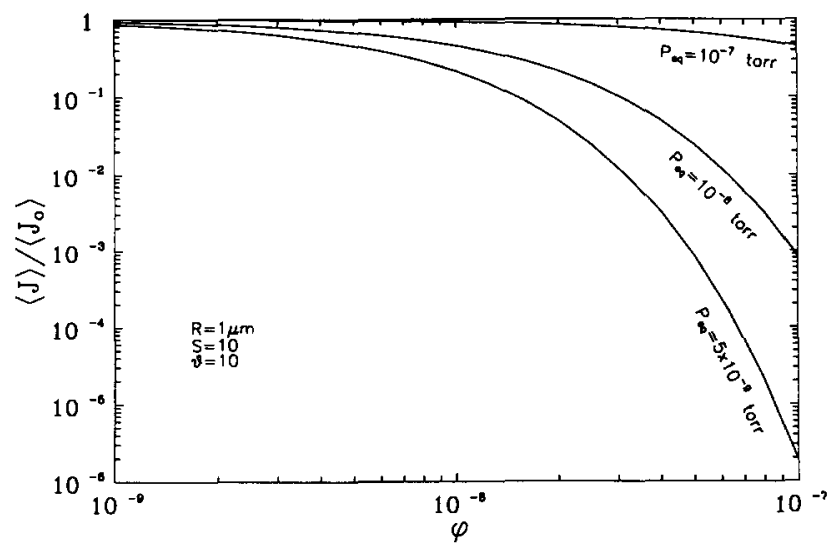

FIG. 2. The effect of cluster scavenging as a function of volume fraction $\phi$.

cles, the normalized nonequilibrium cluster size distribution obeys a complementary error function. In the case with cluster scavenging, $f(g) / n(g)$ changes with $g / g_{*}$ almost exponentially. The value of $-\partial(f / n) / \partial g$ at $x=1$ is related to the rate of nucleation and a smaller slope at this point in the presence of scavenging leads to a lower rate of nucleation.

The reduction in the rate of nucleation due to the cluster scavenging depends on several factors which appears explicitly in Eq. (44). The ratio $\left\langle J\left(g_{*}\right)\right\rangle /\left\langle J_{0}\right\rangle$ is a function of the dimensionless parameters $\epsilon, g_{*}$, and $\eta$. While $g_{*}$ and $\epsilon$ are determined by the dimensionless variables $S$ and $\theta, \eta$ is determined not only by the physical properties of the condensable species, characterized by $\theta, s_{1}$, and $n_{1}$, and the number concentration of sink particles $(\rho)$ and their sizes $(R)$, but also by the molecular number concentration of the background gas $\left(\boldsymbol{n}_{a}\right)$.

The ratio of the nucleation rate predicted from Eq. (44) to the corresponding nucleation rate considering only monomer scavenging is shown as a function of the volume fraction $\phi$ for fixed values of $\theta, S$, and $R$ for different condensing species characterized by $p_{\mathrm{eq}}$ in Fig. 2. The value of $\theta$ chosen for these calculations is 10 which is typical of that for condensable compounds found in the atmosphere $(\theta=6.2$ for water vapor at $T=298 \mathrm{~K}$ ). For atmospheric applications, a typical value of $\phi$ is of the order of $10^{-8}$ taking the number

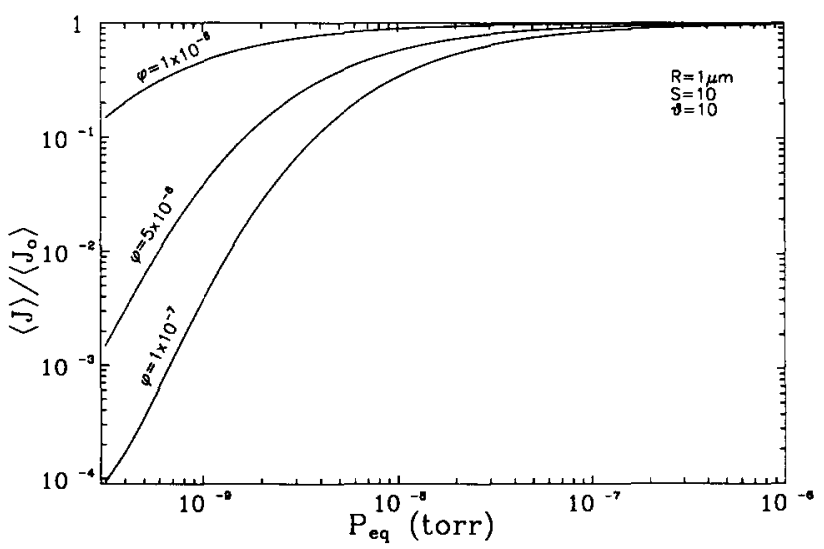

FIG. 3. The effect of cluster scavenging as a function of equilibrium vapor pressure $p_{\mathrm{eq}}$. 


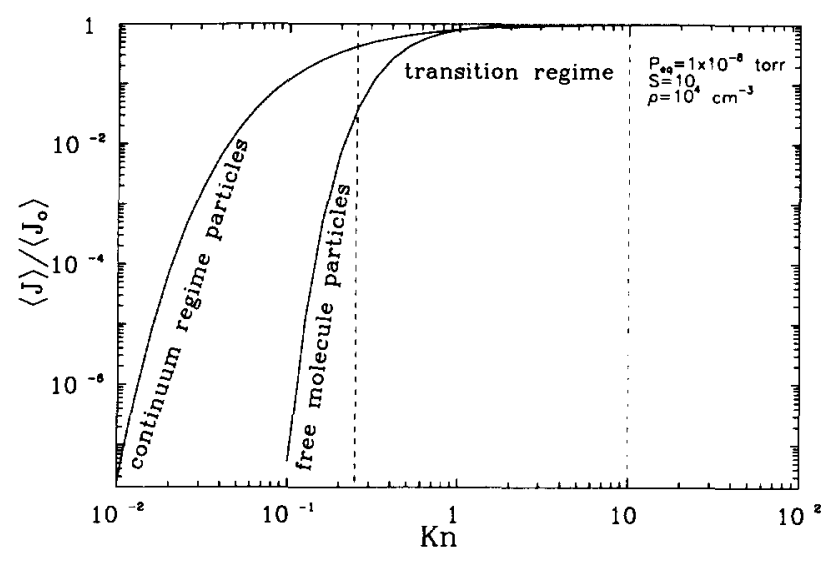

FIG. 4. The effect of cluster scavenging as a function of Knudsen number Kn.

concentration of primary particles as $\rho=10^{4} \mathrm{~cm}^{-3}$ of $1 \mu \mathrm{m}$ in radius. We note that the ratio $\langle J\rangle /\left\langle J_{0}\right\rangle$ decreases as the volume fraction of preexisting particles increases. As in the case of cluster scavenging in the presence of a free molecule aerosol, ${ }^{1}$ cluster scavenging has the most pronounced effect for species with lower vapor pressure. Since $\eta$ increases with decreasing $p_{\text {eq }}$, a larger value of $\eta$ results in a greater depletion of the nonequilibrium number concentration of critical sized clusters, thus a larger reduction in the ratio $\langle J\rangle /\left\langle J_{0}\right\rangle$. This feature is also clearly shown in Fig. 3. From both Figs. 2 and 3 , it can be seen that for typical values of $\phi$ in the atmosphere, the scavenging of prenucleation embryos by any preexisting continuum regime particles of condensible species with vapor pressures higher than about $10^{-7}$ Torr can be generally ignored. Many species, however, that are suggested to be responsible for the formation of new aerosol ${ }^{18}$ are those with vapor pressures lower than this value.

The ratio $\langle J\rangle /\left\langle J_{0}\right\rangle$ is shown as a function of the size of the preexisting particles in Fig. 4 based on the above result and that obtained in Ref. 1 for free molecule particles. Here the Knudsen number is defined as the ratio of the mean free path of the background gas to the radius of the sink particle. For the results shown, the mean free path is calculated by assuming the pressure of the background gas is $1 \mathrm{~atm}$ at $T=298 \mathrm{~K}$. If the transition regime is characterized by the

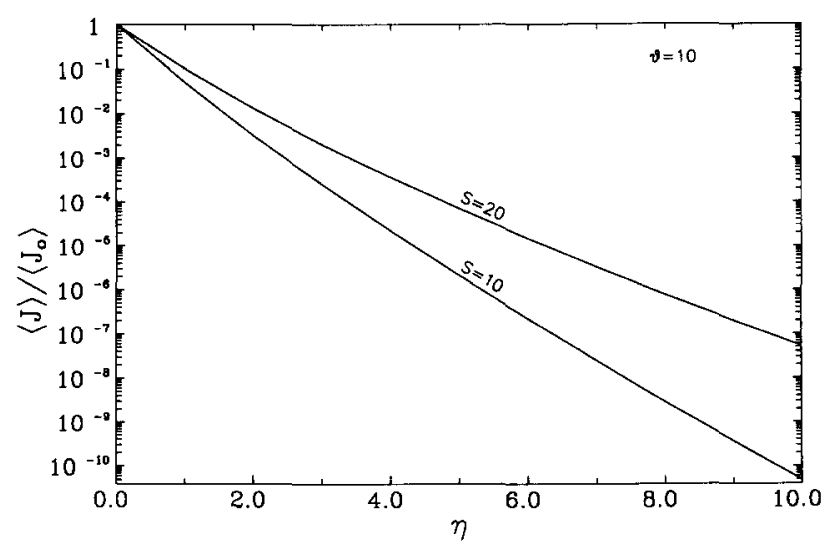

FIG. 5. The effect of cluster scavenging as a function of $\eta$.

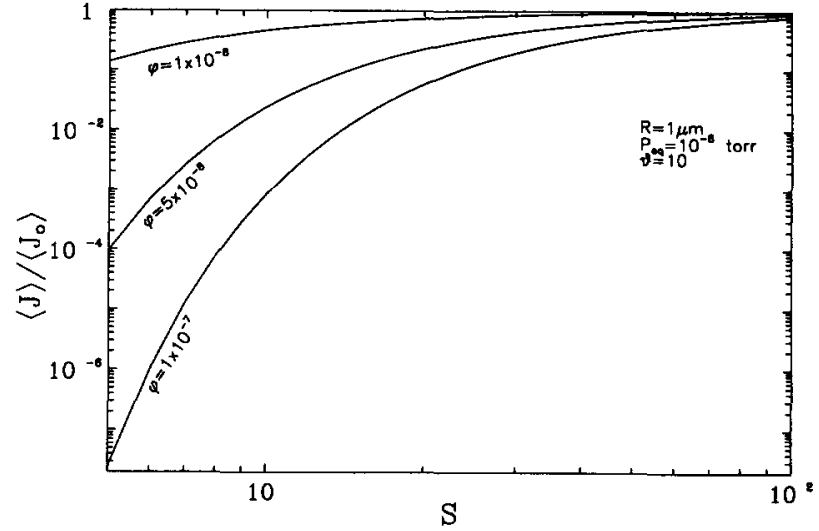

FIG. 6. The effect of cluster scavenging as a function of supersaturation ratio $S$.

value of $\mathrm{Kn}$ in the interval of 0.25 to 10 , the reduction in the nucleation rate due to cluster scavenging by transition regime particles can be estimated by the formula obtained above for continuum regime particles.

In Fig. 5, we plot the ratio $\langle J\rangle /\left\langle J_{0}\right\rangle$ as a function of the dimensionless parameter $\eta$. As discussed above, a larger value of $\eta$ results in a greater depletion of the nonequilibrium number concentration of critical sized clusters, thus a larger reduction in the nucleation rate. The value of $\eta$, as given by Eq. (32) can vary substantially. If there are no preexisting particles, $\eta=0$. For $S=10, \theta=10, p_{\mathrm{eq}}=10^{-8}$ atm, $\rho=10^{4} \mathrm{~cm}^{-3}, R=10^{-4} \mathrm{~cm}$ at $T=298 \mathrm{~K}$ and $1 \mathrm{~atm}$, $\eta \approx 10$. Since $\eta$ is inversely proportional to $n_{a}$, the molecular number concentration of the background gas, a reduction in pressure at a constant concentration of preexisting particles leads to a larger value of $\eta$ and greater effect of cluster scavenging. Indeed, certain experimental nucleation systems involve operation at reduced pressure, such as ones utilizing jet expansion. ${ }^{19}$

An increase in supersaturation lowers the energy barrier and promotes nucleation. In the competition for clusters between the process of nucleation and scavenging by sink particles, it is expected that as $S$ increases, the ratio $\langle J\rangle /\left\langle J_{0}\right\rangle$ becomes larger, as shown in Fig. 6. As $S$ approaches 100 , the ratio approaches unity for $\phi$ in the range of $10^{-8}$ to $10^{-7}$.

The results presented here are applicable to steady-state nucleation in which the characteristic time for change in the saturation ratio is so long compared with the characteristic time to establish a steady-state cluster distribution. For a rapid change in saturation ratio that might occur in certain experimental systems, a general nonstationary solution is still not available. Finally, the system we have considered is taken to be spatial uniform. The effect of spatial inhomogeneities of preexisting particles on the rate of nucleation has yet to be considered.

In summary, a closed-form expression for the influence of cluster scavenging on the rate of homogeneous nucleation of a vapor in the presence of continuum regime particles is obtained by solving the spatially averaged kinetic equation for cluster balance by the method of singular perturbation. The reduction in nucleation rate due to cluster scavenging is shown to be dependent largely on the number concentration, 
size of the sink particles, and the molecular number concentration of the background gas.

\section{ACKNOWLEDGMENT}

This work was supported by National Science Foundation Grant No. ATM-8503103.

${ }^{1}$ G. Shi and J. H. Seinfeld, J. Colloid Interface Sci. (to be published).

${ }^{2}$ A. Pesthy, R. C. Flagan, and J. H. Seinfeld, J. Colloid Interface Sci. 91, 525 (1983).

${ }^{3}$ J. E. Stern, J-J. Wu, R. C. Flagan, and J. H. Seinfeld, J. Colloid Interface Sci. 110, 533 (1986).

${ }^{4}$ R. McGraw and P. H. McMurry, J. Colloid Interface Sci. 92, 584 ( 1983).

${ }^{5}$ K. Binder, Phys. Rev. B 15, 4424 (1977).

${ }^{5}$ K. Binder, Phys. Rev. B 15, 4424 (1977).
${ }^{6}$ F. F. Abraham, Homogeneous Nucleation Theory (Academic, New York, 1974).

${ }^{7}$ M. Muthukumar, J. Chem. Phys. 76, 2667 (1982).

${ }^{8}$ J. A. Marques and J. Ross, J. Chem. Phys. 81, 536 (1984).

${ }^{9}$ M. Fixman, J. Chem. Phys. 81, 3666 (1984).

${ }^{10}$ N. G. van Kampen, Int. J. Quantum Chem. Symp.16, 101 (1982).

${ }^{11} \mathrm{~K}$. Mattern and B. U. Felderhof, Phys. Status Solidi A 143, 1 (1987).

${ }^{12}$ M. Tokuyama and R. I. Cukier, J. Chem. Phys. 76, 6202 (1982).

${ }^{13}$ J. Rubinstein and S. Torquato, J. Chem. Phys. 88, 6372 (1988).

${ }^{14}$ C. W. J. Beenakker and J. Ross, J. Chem. Phys. 84, 3857 (1986).

${ }^{15}$ D. R. Warren and J. H. Seinfeld, J. Colloid Interface Sci. 105, 136 (1985).

${ }^{16}$ D. R. Warren, K. Okuyama, Y. Kousaka, J. H. Seinfeld, and R. C. Flagan, J. Colloid Interface Sci. 116, 562 (1987).

${ }^{17}$ K. Binder, Rep. Prog. Phys. 50, 783 (1987).

${ }^{18} \mathrm{~J}$. H. Seinfeld, Science 243, 745 (1989).

${ }^{19}$ D. Kotake and I. I. Glass, Prog. Aerospace Sci. 19, 129 (1981). 